\title{
BMJ Open Association between proprotein convertase subtilisin/kexin type 9 and late saphenous vein graft disease after coronary artery bypass grafting: a cross- sectional study
}

Jing Gao, ${ }^{1,2}$ Hai-Bo Wang, ${ }^{3}$ Jian-yong Xiao, ${ }^{4}$ Min Ren, ${ }^{2}$ Kathleen Heather Reilly, ${ }^{5}$ Yu-Ming Li, ${ }^{1}$ Yin Liu ${ }^{4}$

To cite: Gao J, Wang H-B, Xiao J, et al. Association between proprotein convertase subtilisin/kexin type 9 and late saphenous vein graft disease after coronary artery bypass grafting: a crosssectional study. BMJ Open 2018;8:e021951. doi:10.1136/ bmjopen-2018-021951

\section{- Prepublication history for} this paper is available online. To view these files, please visit the journal online (http://dx.doi. org/10.1136/bmjopen-2018021951).

JG and H-BW contributed equally.

Received 30 January 2018 Revised 7 May 2018 Accepted 8 June 2018

Check for updates

(C) Author(s) (or their employer(s)) 2018. Re-use permitted under CC BY-NC. No commercial re-use. See rights and permissions. Published by BMJ.

For numbered affiliations see end of article.

Correspondence to Professor Yu-Ming Li; cardiolab@live.com and Professor Yin Liu; liuyin2088@163.com

\section{ABSTRACT}

Objective The study aims to explore the association between serum proprotein convertase subtilisin/kexin type 9 (PCSK9) level and saphenous vein grafts disease (SVGD) after coronary artery bypass grafting (CABG).

Design A cross-sectional study.

Setting A secondary hospital in Tianjin City, China.

Participants A total of 231 participants were included in the study. Inclusion criteria were as follows: age $\geq 18$ years, previous CABG surgery at least 12 months ago, at least one SVG for bypass during CABG, abnormal non-invasive test results or recurrent stable angina pectoris by coronary angiography indications, and willing to participate and sign informed consent. Participants with any of the following were excluded from the study: congenital valvular disease, decompensated heart failure, anaemia defined as a haemoglobin level of $<12 \mathrm{~g} / \mathrm{dL}$ in women or $<13 \mathrm{~g} / \mathrm{dL}$ in men, malignant neoplasms, renal failure, severe hepatic disease, thyroid disease, acute or chronic inflammatory disease and chronic obstructive lung disease.

Primary outcome measure SVGD was defined as at least one SVG with significant stenosis $(\geq 50 \%)$. Circulating PCSK9 levels were measured using commercial ELISA kits according to the manufacturer's instructions.

Results The mean PCSK9 level in the SVGD group was significantly higher than that in the patent group ( $275.2 \pm 38.6$ vs $249.3 \pm 37.7, p<0.01)$. The multivariate logistic regression model revealed a significant association between serum PCSK9 and SVGD (OR 2.08, 95\% Cl 1.462.95) per 1 SD increase in serum PCSK9.

Conclusions The present study is the first to identify an independent association between PCSK9 and late SVGD after adjustment for established cardiovascular risk factors. A multicentre prospective cohort study with large sample size should be conducted in the future to further research this relationship.

\section{INTRODUCTION}

Coronary artery disease (CAD) is the leading cause of mortality worldwide. ${ }^{1}$ Coronary artery bypass grafting (CABG) can prolong life in patients with severe CAD and is a
Strengths and limitations of this study

- The present study is the first to identify an independent association between proprotein convertase subtilisin/kexin type 9 (PCSK9) level and late saphenous vein grafts disease in Chinese patients.

- The participants who had coronary artery bypass grafting surgery at least 12 months ago were enrolled to minimise the impact of graft failure factors.

- The relationships between PCSK9 and blood-lipid levels were extensively explored in this study.

- This was a cross-sectional study in a single centre which could not confirm temporality or generalisability.

common treatment for CAD. ${ }^{2}$ Both arterial and saphenous vein grafts (SVGs) are currently used in CABG surgery. SVGs are still frequently used, although arterial grafts have a high long-term patency rate. ${ }^{3}$ SVG disease (SVGD) is a frequent problem and limits the benefits of CABG, with graft patency $89 \%$ at 1 year, dropping to $61 \%$ after 10 years. ${ }^{3}$ SVGD development can be classified into three phases: thrombosis (first month), intimal hyperplasia (1-12 months) and atherosclerosis (>12 months). ${ }^{4}$ In the latter stages, atherosclerosis becomes the most important cause of late graft failure after the first year. ${ }^{5}$

The pathogenesis of atherosclerosis is multifactorial and hyperlipidaemia is associated with long-term outcome after $\mathrm{CABG}^{6}{ }^{6}$ An aggressive lipid-lowering therapy has been shown to significantly delay the progression of atherosclerosis in vein grafts after $\mathrm{CABG}^{7}$ Patients treated with aggressive lipid-lowering therapy had significant lower risk of substantial lesion progression $(\geq 0.4 \mathrm{~mm}$ reduction in lumen diameter at the site of the lesion) in the left main coronary artery compared with 
moderate therapy $(13.8 \%$ vs24.1\%, $\mathrm{p}=0.008) .{ }^{7}$ Another study showed that an aggressive lowering of low-density lipoprotein cholesterol (LDL-C) levels to $<100 \mathrm{mg} / \mathrm{dL}$ delayed the progression of atherosclerosis in grafts. ${ }^{8}$ The aggressive lipid-lowering strategy was also recommended in European Society of Cardiology/American Heart Association guidelines. ${ }^{9}$ Proprotein convertase subtilisin/ kexin type 9 (PCSK9) is a circulating serine protease that has received considerable attention as a promising target for lipid-lowering therapy. ${ }^{11}$ PCSK9 can efficiently bind to LDL receptors and promotes intracellular degradation of the LDL receptor, resulting in increased serum LDL-C concentration by reducing the uptake of LDL-C. ${ }^{12}{ }^{13} \mathrm{~A}$ study found that a loss-of-function mutation in PCSK9 was associated with low LDL-C levels and protective against CAD. ${ }^{14}$ Several randomised controlled trials have shown that PCSK9 inhibitors can decrease LDL-C levels, resulting in reduction of cardiovascular events without major adverse effects. ${ }^{1516}$ Some studies that have investigated the association of serum PCSK9 with cardiovascular risk have indicated that serum PCSK9 could independently predict cardiovascular events in patients with stable CAD. ${ }^{17-20}$

As CAD and SVGD have similar pathophysiological mechanisms, we hypothesised that serum PCSK9 levels might be associated with the development of SVGD. The relationship between serum PCSK9 and SVGD has not been previously explored. Thus, we aimed to assess the association between serum PCSK9 levels and the risk of SVGD occurrence.

\section{METHODS}

\section{Study population}

In this cross-sectional study, we enrolled 231 patients that had undergone CABG surgery $\geq 12$ months ago between October 2015 and December 2016 at the Cardiology Department of Tianjin Chest Hospital.

Inclusion criteria were as follows: age $\geq 18$ years, previous CABG surgery at least 12 months ago, at least one SVG for bypass during CABG, abnormal non-invasive test results or recurrent stable angina pectoris by coronary angiography indications, willing to participate and sign informed consent. Participants with any of the following were excluded from the study: congenital valvular disease, decompensated heart failure, anaemia defined as a haemoglobin level of $<12 \mathrm{~g} / \mathrm{dL}$ in women or $<13 \mathrm{~g} / \mathrm{dL}$ in men according to WHO, malignant neoplasms, renal failure, severe hepatic disease, thyroid disease, acute or chronic inflammatory disease and chronic obstructive lung disease. Patients who underwent $\mathrm{CABG}<12$ months before enrolment were also excluded.

\section{DATA COLLECTION}

After receiving written informed consent, demographic and clinical characteristics and fasting blood samples were collected from all participants. Clinical staff reviewed medical records and extracted participants' clinical information. Cardiac function class was determined according to the New York Heart Association (NYHA) functional classification. Mean systolic blood pressure (SBP) and diastolic blood pressure (DBP) in sitting state was measured by one physician. Participants were asked to fast from midnight the night before. Blood samples were collected from antecubital veins before coronary angiography. Venous blood samples were drawn into EDTAcoated tubes, promptly centrifuged and stored at $-80^{\circ} \mathrm{C}$ until analysis. Circulating PCSK9 levels were measured using commercial ELISA kits (R\&D Systems, Minnesota, USA) according to the manufacturer's instructions. Total cholesterol, triglyceride, high-density lipoprotein cholesterol (HDL-C), LDL-C, very low-density lipoprotein (VLDL), lipoprotein (a), apolipoprotein A, apolipoprotein $\mathrm{B}$, fasting glucose and homocysteinaemia were measured enzymatically using an autoanalyzer (Hitachi 911 autoanalyzer; Hitachi, Tokyo, Japan). A haematology analyzer (Sysmex XE-2001, Sysmex, Kobe, Japan) was used to determine the blood count, including the platelet count and haemoglobin levels.

Hypertension was defined as $\mathrm{SBP} \geq 140 \mathrm{~mm} \mathrm{Hg}$ and/ or DBP $\geq 90 \mathrm{~mm} \mathrm{Hg}$, or use of antihypertensive drugs. Diabetes mellitus was diagnosed as a fasting serum glucose level $\geq 7.0 \mathrm{mmol} / \mathrm{L}$, a self-reported history of diabetes mellitus or active use of any hypoglycaemic drugs. Hypercholesterolaemia was considered present if at least one of three of the following criteria was met: fasting total cholesterol $\geq 6.45 \mathrm{mmol} / \mathrm{L}$, self-reported hyperlipidaemia and self-reported use of lipid-lowering drugs. Participants who reported that they smoked regularly were classified as current smokers.

Coronary angiography was performed using the Judkins technique according to standard clinical practice. The SVGs were displayed in multiple plane images and the degree of stenosis was determined in the plane image showing the most severe narrowing. All angiograms were evaluated by at least two interventional cardiologists who were blinded to patients' characteristics. The SVGD was defined as at least one SVG with significant stenosis $(\geq 50 \%)$ by both cardiologists, and the participants were divided into two groups according to SVGD status.

\section{STATISTICAL ANALYSIS}

Statistical tests were performed using SAS V.9.3 software (Cary, North Carolina, USA). Results were presented as mean \pm SD or median \pm IQR for continuous variables, and frequency (percentage) for categorical variables. The differences of clinical characteristics and biochemical parameters between the two groups were compared using Student's $t$-test (or Mann-Whitney U-test) and $\chi^{2}$ test/ Fisher's exact test as appropriate. The crude and adjusted associations between PCSK9 and SVGD were evaluated using univariate and multivariate logistic regression models and are presented as ORs and 95\% CIs. Variables found to be significant in univariate analysis $(p<0.20)$ were 
considered for inclusion in the final multivariate model; stepwise selection was used to determine the final model with entry criteria of $\mathrm{p}=0.20$ and stay criteria of $\mathrm{p}=0.05$. To increase statistical power, we estimated the effect of continuous PSCK9 on SVGD risk per 1 SD increase. The correlations between PCSK9 and other clinical characteristics of participants were evaluated using the Pearson correlation coefficient. All tests were two-sided and $p$ value $<0.05$ was considered statistically significant for all analysis.

\section{Patient and public involvement}

Patients were not involved in the development of the research questions, design, recruitment or implementation of the study. Major findings from the study will be disseminated through national conference posters and social media. In addition, with the contact information collected during study, the participants will be notified of the study results through telephone or by post.

\section{RESULTS}

The 231 consecutively enrolled participants were divided into two groups: 124 (53.7\%) patients with SVGD and 107 (46.3\%) patients with patent SVGs (patent group). The demographic and clinical characteristics of the participants by SVGD status are listed in table 1 . These participants were predominantly male $(80.9 \%)$ with a mean age of $65.6 \pm 8.2$ years old. There were no significant differences between the two groups regarding demographical characteristics. The mean time from CABG surgery to the last coronary angiogram was significantly longer in the SVGD group $(8.1 \pm 3.8$ vs $6.1 \pm 3.7$ years, $p<0.01)$. Compared with participants without SVGD, the participants in the SVGD group were younger at the time of their CABG operation and were more likely to have a family history of cardiovascular disease. The SVGD participants were also more likely to report smoking, had higher left ventricular end-diastolic diameter (LVEDD) and more likely to have acute coronary syndrome.

The laboratory results of participants by SVGD status are presented in table 2. The participants in the SVGD group had higher levels of triglycerides, VLDL, lipoprotein (a) and PCSK9 (figure 1). There were no statistically significant differences for other laboratory variables. The mean value of PCSK9 was $263.2 \pm 40.2 \mathrm{ng} / \mathrm{mL}$ among all participants, and the mean PCSK9 value in the SVGD group was significantly higher than that in patent group $(275.2 \pm 38.6$ vs $249.3 \pm 37.7, \mathrm{p}<0.01)$. There was also a linear trend between serum PCSK9 quartiles and SVGD ( $p$ for trend $<0.001$ ).

The univariate logistic regression model revealed a significant association between serum PCSK9 and SVGD (OR 2.10, 95\% CI 1.53 to 2.87) per $1 \mathrm{SD}$ increase in serum PCSK9. Serum PCSK9 concentration was additionally categorised by quartiles to investigate the consistency of its association with SVGD across quartiles. With quartile 1 serum PCSK9 as reference, the ORs for quartiles 2-4 were 1.25 (95\% CI 0.59 to 2.66 ), 4.87 (95\% CI 2.21 to 10.75 ) and 4.22 (95\% CI 1.94 to 9.18). The association was attenuated, but remained significant after adjustment for other established cardiovascular risk factors (table 3), and the OR for 1 SD increase of serum PCSK9 was 1.83 (95\% CI 1.29 to 2.59). In addition to serum PCSK9 level, the following variables remained statistically significant in the multivariate logistic regression model: time from CABG surgery, family history of cardiovascular disease, ischaemia type, native lesion vessel number and VLDL.

Non-lipid cardiovascular risk factors did not have significant impact on serum PCSK9 levels. The Pearson correlation coefficients between PCSK9 and age $(\mathrm{r}=-0.09$, $\mathrm{p}=0.19)$, time from CABG surgery $(\mathrm{r}=0.04, \mathrm{p}=0.52)$, SBP $(\mathrm{r}=-0.01, \mathrm{p}=0.90)$ and $\mathrm{DBP}(\mathrm{r}=0.06, \mathrm{p}=0.33)$ were all quite small (table 4). In contrast, serum PCSK9 levels correlated modestly with white blood cell counts $(r=0.25, p<0.01)$ and VLDL $(\mathrm{r}=0.18, \mathrm{p}<0.01)$. There were no statistically significant correlations between serum PCSK9 levels and other lipid parameters.

\section{DISCUSSION}

To the best of our knowledge, this was the first study to determine the association between serum PSCK9 and late SVGD after CABG. The mean PCSK9 value was significantly higher in patients with SVGD than in patients with patent SVG $(275.2 \pm 38.6$ vs $249.3 \pm 37.7, \mathrm{p}<0.01)$. Moreover, multivariate logistic regression analysis showed that serum PSCK9 level was independently associated with late SVGD.

CABG is a widely performed surgical procedure for $\mathrm{CAD}$ and confers both quality of life and survival benefits to patients. ${ }^{21}$ However, the effectiveness of CABG is weakened by SVGD after CABG, with graft patency dropping to $61 \%$ after 10 years. ${ }^{3}$ Thrombosis, intimal hyperplasia and atherosclerosis are the main causes of SVGD according to the time period after CABG surgery. ${ }^{4}$ Atherosclerosis is one of the main pathogenic mechanisms for late SVGD 12 months after surgery. ${ }^{22}$ We included participants who had CABG surgery at least 12 months ago in our study to minimise the graft failure factors related to the surgery itself. Accordingly, the mean time from surgery was $8.1 \pm 3.8$ years in the SVGD group and $6.1 \pm 3.7$ years in the patent group.

The relationship between PCSK9 function and CAD development and prognosis has been comprehensively investigated over the past decades. ${ }^{13}$ In a prospective cohort study, 4232 participants were enrolled to investigate the relationship between PCSK9 and incident cardiovascular events. ${ }^{17}$ During the 15 years of follow-up, baseline PCSK9 concentration predicted incident cardiovascular events. Concentration in quartile 4 compared with quartile 1 was independently associated with an HR of 1.48 (95\% CI 1.12 to 1.95) after adjustment for established cardiovascular risk factors. ${ }^{17}$ Due to discrepant results in evaluating PCSK9 as a biomarker of cardiovascular risk, a systematic review was performed, showing that 
Open access

Table 1 Demographic, clinical characteristics of participants and association with saphenous vein graft disease (SVGD)

\begin{tabular}{|c|c|c|c|}
\hline Variable & $\begin{array}{l}\text { Participants with SVGD } \\
(\mathrm{n}=124)\end{array}$ & $\begin{array}{l}\text { Participants without SVGD } \\
(n=107)\end{array}$ & $P$ values \\
\hline Age (years) & $65.0 \pm 8.5$ & $66.2 \pm 7.9$ & 0.26 \\
\hline Male, n (\%) & $100(81.3)$ & $86(80.4)$ & 0.86 \\
\hline BMI $\left(\mathrm{kg} / \mathrm{m}^{2}\right)$ & $25.3 \pm 4.1$ & $27.3 \pm 1.9$ & 0.15 \\
\hline CABG age (years) & $56.9 \pm 9.1$ & $60.2 \pm 8.0$ & $<0.01$ \\
\hline Time from CABG surgery (years) & $8.1 \pm 3.8$ & $6.1 \pm 3.7$ & $<0.01$ \\
\hline $\begin{array}{l}\text { Family history of cardiovascular disease, } \\
\mathrm{n}(\%)\end{array}$ & $37(29.8)$ & $13(12.2)$ & $<0.01$ \\
\hline Hypertension, n (\%) & $92(74.2)$ & $68(63.6)$ & 0.08 \\
\hline Diabetes mellitus, n (\%) & $52(41.9)$ & $53(49.5)$ & 0.25 \\
\hline Hypercholesterolaemia, n (\%) & $86(69.4)$ & $67(62.6)$ & 0.28 \\
\hline History of stroke, n (\%) & $22(17.7)$ & $13(12.1)$ & 0.24 \\
\hline Current smoking, n (\%) & $71(57.3)$ & $45(42.1)$ & 0.02 \\
\hline Current alcohol drinking, $\mathrm{n}(\%)$ & $38(30.7)$ & $29(27.1)$ & 0.55 \\
\hline \multicolumn{4}{|l|}{ Cardiac function (NYHA classes), n (\%) } \\
\hline$|-| \mid$ & $102(82.3)$ & $82(76.6)$ & 0.29 \\
\hline III-IV & $22(17.7)$ & $25(23.4)$ & \\
\hline \multicolumn{4}{|l|}{ Ischaemia type, n (\%) } \\
\hline Stable angina & $18(14.5)$ & $31(29.0)$ & $<0.01$ \\
\hline Acute coronary syndrome & $106(85.5)$ & $76(71.0)$ & \\
\hline \multicolumn{4}{|l|}{ Native lesion vessel number } \\
\hline 1 & $1(0.8)$ & $6(5.6)$ & 0.04 \\
\hline 2 & $29(23.6)$ & $33(30.8)$ & \\
\hline 3 & 93 (75.6) & $68(63.6)$ & \\
\hline Progression of native vessels, $\mathrm{n}(\%)$ & $120(96.8)$ & $98(91.6)$ & 0.09 \\
\hline Number of SVG (mean) & $2.7 \pm 0.7$ & $2.6 \pm 0.7$ & 0.08 \\
\hline \multicolumn{4}{|l|}{ Graft types } \\
\hline SVG-LAD, n (\%) & $35(34.0)$ & $35(58.3)$ & $<0.01$ \\
\hline SVG-RCA, n (\%) & $31(29.8)$ & $13(27.1)$ & 0.73 \\
\hline SVG-LCX, n (\%) & $25(23.8)$ & $16(30.2)$ & 0.39 \\
\hline SVG-Diag, n (\%) & $37(33.9)$ & $29(49.2)$ & 0.05 \\
\hline LAD-PDA, n (\%) & $70(59.8)$ & $66(77.7)$ & $<0.01$ \\
\hline LCX-OM, n(\%) & $51(45.5)$ & 38 (61.3) & 0.04 \\
\hline \multicolumn{4}{|l|}{ Stenosis degree of SVG } \\
\hline SVG-LAD & $67.1 \pm 43.4$ & $5.8 \pm 3.6$ & $<0.01$ \\
\hline SVG-RCA & $78.4 \pm 39.5$ & $7.5 \pm 3.1$ & $<0.01$ \\
\hline SVG-LCX & $70.5 \pm 40.9$ & $6.0 \pm 3.4$ & $<0.01$ \\
\hline SVG-Diag & $66.4 \pm 47.1$ & $13.2 \pm 3.5$ & $<0.01$ \\
\hline SVG-PDA & $68.5 \pm 40.3$ & $14.1 \pm 3.0$ & $<0.01$ \\
\hline SVG-OM & $74.5 \pm 38.0$ & $17.5 \pm 4.2$ & $<0.01$ \\
\hline Ejection fraction, $\mathrm{n}(\%)$ & $54.2 \pm 9.1$ & $55.8 \pm 8.2$ & 0.19 \\
\hline LVEDD (mm) & $53.1 \pm 5.7$ & $50.9 \pm 7.3$ & 0.01 \\
\hline \multicolumn{4}{|l|}{ Drug usage, n (\%) } \\
\hline Aspirin, n (\%) & $118(95.2)$ & $102(95.3)$ & 0.85 \\
\hline$\beta$-Blocker, n (\%) & 87 (70.2) & 83 (77.6) & 0.52 \\
\hline
\end{tabular}

Continued 
Table 1 Continued

\begin{tabular}{llll}
\hline Variable & $\begin{array}{l}\text { Participants with SVGD } \\
(\mathbf{n = 1 2 4})\end{array}$ & $\begin{array}{l}\text { Participants without SVGD } \\
(\mathbf{n = 1 0 7 )}\end{array}$ & P values \\
\hline Statin, $\mathrm{n}(\%)$ & $94(75.8)$ & $78(72.9)$ & 0.73 \\
ACE/ARB, $\mathrm{n}(\%)$ & $84(67.7)$ & $71(66.4)$ & 0.70 \\
\hline Clopidogrel, $\mathrm{n}(\%)$ & $62(50.0)$ & $65(60.7)$ & 0.10 \\
\hline
\end{tabular}

BMI, body mass index; CABG, coronary artery bypass grafting; LAD, left anterior descend; LCX, left circumflex artery; LVEDD, left ventricular end-diastolic diameter; NYHA, New York Heart Association; OM, obtuse marginal branch; PDA, posterior descend artery; RCA, right coronary artery; UA, uric acid.

PCSK9 levels were modestly, but significantly correlated with risk of cardiovascular events. ${ }^{23}$ An intervention trial showed that PCSK9 levels increased with improvements in fitness and visceral fat mobilisation after the intervention (improving dietary quality and increasing physical activity) among patients undergoing CABG surgery. ${ }^{24}$

PCSK9 impacts atherosclerosis through both lipid and non-lipid pathways. Chan et al found an independent association between PCSK9 and carotid intimamedia wall thickness after adjustment for traditional cardiovascular risk factors. ${ }^{25}$ Comprehensive reviews have described the key role of PCSK9 in LDL-C metabolism by direct interaction with LDL receptors and ultimately plasm LDL-C. ${ }^{26}{ }^{27}$ Gain-of-function mutations in PCSK9 resulted in higher levels of LDL-C, whereas loss-of-function PCSK9 sequence variations were found to be associated with

Table 2 Laboratory testing results of participants and association with saphenous vein graft disease (SVGD)

\begin{tabular}{|c|c|c|c|}
\hline Variable & $\begin{array}{l}\text { Participants with SVGD } \\
(n=124)\end{array}$ & $\begin{array}{l}\text { Participants without SVGD } \\
(n=107)\end{array}$ & $P$ values \\
\hline White blood cell count $\left(10^{9} / \mathrm{L}\right)$ & $6.9 \pm 2.0$ & $6.6 \pm 1.5$ & 0.29 \\
\hline Red blood cell count $\left(10^{12} / \mathrm{L}\right)$ & $4.6 \pm 0.6$ & $5.8 \pm 11.9$ & 0.31 \\
\hline Haemoglobin (g/L) & $137.7 \pm 19.2$ & $138.3 \pm 19.8$ & 0.80 \\
\hline Platelet count $\left(10^{9} / \mathrm{L}\right)$ & $204.7 \pm 58.8$ & $201.4 \pm 43.1$ & 0.63 \\
\hline Red cell distribution width (\%) & $12.5 \pm 1.0$ & $13.8 \pm 1.1$ & 0.24 \\
\hline Mean platelet volume & $12.9 \pm 12.4$ & $10.2 \pm 10.3$ & 0.35 \\
\hline Plateletcrit & $0.4 \pm 1.5$ & $0.2 \pm 0.1$ & 0.15 \\
\hline Platelet distribution width & $15.2 \pm 2.8$ & $15.2 \pm 2.7$ & 0.82 \\
\hline Neutrophil-to-lymphocyte ratio & $3.3 \pm 2.1$ & $3.0 \pm 1.2$ & 0.20 \\
\hline Platelet-to-lymphocyte ratio & $140.4 \pm 61.9$ & $135.1 \pm 47.3$ & 0.46 \\
\hline Total cholesterol (mmol/L) & $4.5 \pm 1.2$ & $4.3 \pm 1.2$ & 0.10 \\
\hline Triglycerides (mmol/L) & $1.9 \pm 1.1$ & $1.6 \pm 0.8$ & 0.04 \\
\hline $\mathrm{HDL-C}(\mathrm{mmol} / \mathrm{L})$ & $1.0 \pm 0.4$ & $1.0 \pm 0.3$ & 0.63 \\
\hline LDL-C (mmol/L) & $2.9 \pm 1.1$ & $2.8 \pm 1.0$ & 0.41 \\
\hline VLDL (mmol/L) & $0.6 \pm 0.3$ & $0.5 \pm 0.2$ & $<0.01$ \\
\hline Lipoprotein (a) (mmol/L) & $83.9 \pm 103.0$ & $59.6 \pm 74.1$ & 0.04 \\
\hline Apolipoprotein A (mmol/L) & $1.1 \pm 0.2$ & $1.1 \pm 0.2$ & 0.86 \\
\hline Apolipoprotein B (mmol/L) & $1.0 \pm 0.3$ & $1.0 \pm 0.3$ & 0.14 \\
\hline Fasting glucose (mmol/L) & $7.0 \pm 5.4$ & $6.4 \pm 2.8$ & 0.30 \\
\hline Homocysteinaemia $(\mu \mathrm{mol} / \mathrm{L})$ & $16.2 \pm 8.4$ & $15.4 \pm 6.7$ & 0.41 \\
\hline $\mathrm{UA}(\mu \mathrm{mol} / \mathrm{L})$ & $338.6 \pm 91.8$ & $344.4 \pm 97.5$ & 0.65 \\
\hline PCSK9 (ng/mL) & $275.2 \pm 38.6$ & $249.3 \pm 37.7$ & $<0.01$ \\
\hline Quartile 1, $\leq 235.51 \mathrm{ng} / \mathrm{mL}$ & $20(16.1)$ & $38(35.5)$ & $<0.01$ \\
\hline Quartile 2, $>235.51$ to $\leq 254.89 \mathrm{ng} / \mathrm{mL}$ & $23(18.5)$ & $35(32.7)$ & \\
\hline Quartile $3,>254.89$ to $\leq 290.13 \mathrm{ng} / \mathrm{mL}$ & $41(33.1)$ & $16(15.0)$ & \\
\hline Quartile 4, >290.13ng/mL & 40 (32.3) & $18(16.8)$ & \\
\hline
\end{tabular}

HDL-C, high-density lipoprotein cholesterol; LDL-C, low-density lipoprotein cholesterol; PCSK9, proprotein convertase subtilisin/kexin type 9; SVGD indicated saphenous vein graft disease; VLDL, very low-density lipoprotein. 


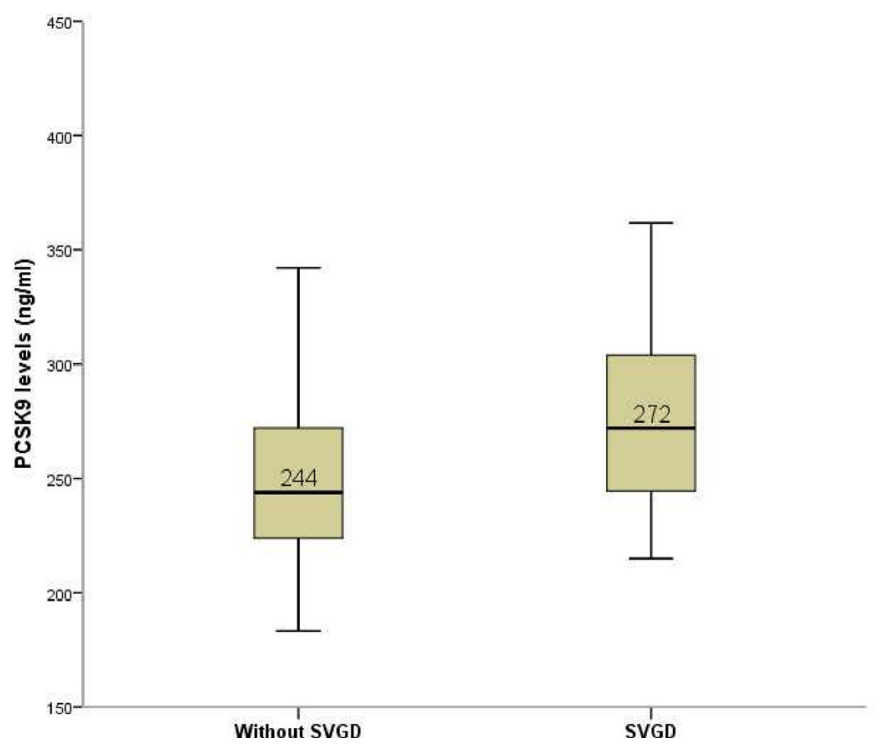

Figure 1 Comparison of proprotein convertase subtilisin/ kexin type 9 (PCSK9) levels between patent group and saphenous vein graft disease (SVGD) group. The mean PCSK9 level in the SVGD group was significantly higher than that in the patent group $(p<0.01)$.

reductions in both LDL-C and cardiovascular risk. ${ }^{13} 14$ However, there was no significant difference in LDL-C levels between subjects with or without SVGD, although an independent association between serum PSCK9 levels and late SVGD has been established. It is speculated that two causes may contribute to the contrary findings. First, lipid-lowering therapy was widely used to control lipid level among patients who underwent CABG surgery, as shown in our study, about $75 \%$ of participants routinely take statins. Second, in addition to induced degradation of LDL receptors and hypercholesterolaemia, PCSK9 may promote atherosclerosis development independent of its impact on lipids. The association between elevated PCSK9

Table 3 Multivariate logistic regression analysis of factors associated with saphenous vein graft disease among patients with coronary artery bypass grafting (CABG)

\begin{tabular}{llc}
\hline Risk factors & OR $(95 \%$ Cl) & P values \\
\hline $\begin{array}{l}\text { Time from CABG surgery } \\
\text { (years) }\end{array}$ & $1.18(1.08$ to 1.29$)$ & $<0.01$ \\
$\begin{array}{l}\text { Family history of } \\
\text { cardiovascular disease }\end{array}$ & $2.38(1.08$ to 5.26$)$ & 0.03 \\
$\begin{array}{l}\text { Ischaemia type (acute } \\
\text { coronary syndrome vs } \\
\text { stable angina) }\end{array}$ & $3.07(1.43$ to 6.57$)$ & $<0.01$ \\
$\begin{array}{l}\text { Native lesion vessel } \\
\text { number }\end{array}$ & $2.49(1.37$ to 4.51$)$ & $<0.01$ \\
$\begin{array}{l}\text { VLDL } \\
\text { Lipoprotein (a) }\end{array}$ & $1.005(1.001$ to 1.009$)$ & 0.04 \\
\hline PCSK9 1 SD increase & $1.83(1.29$ to 2.59$)$ & $<0.01$ \\
\hline
\end{tabular}

PCSK9, proprotein convertase subtilisin/kexin type 9; VLDL, very low-density lipoprotein.
Table 4 Correlations between proprotein convertase subtilisin/kexin type 9 (PCSK9) and other clinical characteristics of participants

\begin{tabular}{lcc}
\hline & PCSK9 & \\
\cline { 2 - 3 } Variables & $\begin{array}{l}\text { Correlation } \\
\text { coefficient }\end{array}$ & P values \\
\hline Age (years) & -0.0870 & 0.19 \\
\hline Time from CABG surgery & 0.0430 & 0.52 \\
\hline SBP (mm Hg) & 0.0085 & 0.90 \\
\hline DBP (mm Hg) & 0.0639 & 0.33 \\
\hline White blood cell count (10 $\left.{ }^{\circ} / \mathrm{L}\right)$ & 0.2533 & $<0.01$ \\
\hline Platelet count (10 $\left.{ }^{\circ} / \mathrm{L}\right)$ & 0.1235 & 0.06 \\
\hline Red cell distribution width $(\%)$ & 0.0430 & 0.52 \\
\hline Mean platelet volume & 0.0805 & 0.23 \\
\hline Plateletcrit & 0.0850 & 0.20 \\
\hline Platelet distribution width & -0.0283 & 0.67 \\
\hline Total cholesterol (mmol/L) & 0.0461 & 0.49 \\
\hline Triglycerides (mmol/L) & 0.1215 & 0.07 \\
\hline HDL-C (mmol/L) & -0.0324 & 0.63 \\
\hline LDL-C (mmol/L) & 0.0178 & 0.79 \\
\hline VLDL (mmol/L) & 0.1772 & $<0.01$ \\
\hline Lipoprotein (a) (mmol/L) & -0.0284 & 0.67 \\
\hline Apolipoprotein A (mmol/L) & -0.0201 & 0.76 \\
\hline Apolipoprotein B (mmol/L) & 0.0763 & 0.26 \\
\hline Fasting glucose (mmol/L) & 0.0177 & 0.79 \\
\hline Homocysteinaemia ( $\mu$ mol/L) & 0.0399 & 0.55 \\
\hline & & \\
\hline
\end{tabular}

DBP, diastolic blood pressure; HDL-C, high-density lipoprotein cholesterol; LDL-C, low-density lipoprotein cholesterol; SBP, indicated systolic blood pressure; VLDL, very low-density lipoprotein.

and cardiovascular risk existed in the absence of elevated LDL, demonstrating the effects of PCSK9 by non-lipid pathways. It has been shown that an inflammatory mechanism plays an important role in atherosclerosis progression. $^{28}{ }^{29}$ Inflammatory biomarkers such as C-reactive protein, interleukin-6 and fibrinogen have been shown to be predictors of cardiovascular events. ${ }^{30}{ }^{31}$ LOX-1 is an important receptor for oxidized-LDL and its expression is upregulated in the inflammatory state. The interaction between LOX-1 activation and PCSK9 may facilitate atherogenesis, particularly in an inflammatory state. ${ }^{32}$

Serum PCSK9 was found to be positively correlated with VLDL. In a double-blind randomised-controlled trial, it was speculated that PCSK9 played a role in regulating VLDL metabolism as the study found that serum PCSK9 reduction correlated with decreases in VLDL concentration among patients with type 2 diabetes who receive fenofibrate treatment. ${ }^{33}$ LDL receptors can promote intracellular degradation of apolipoprotein B as they bind to nascent apolipoprotein B during the course of VLDL assembly. ${ }^{34}$ VLDL secretion might be stimulated by PCSK9 through degradation of LDL receptors. ${ }^{35}{ }^{36} \mathrm{It}$ 
has been postulated that PCSK9 binding to LDL receptors can limit apolipoprotein B from intracellular catabolism induced by LDL receptors. ${ }^{37}$ In addition to exerting effects from LDL receptors, PCSK9 also interferes with intracellular transport and degradation of VLDL receptors. ${ }^{38}$ The absence of circulating PCSK9 has been shown to lead to increased levels of surface VLDL receptors in the perigonadal tissue. ${ }^{39}$

Our data showed that serum PCSK9 levels correlated modestly with white blood cell counts. Another study also found that PCSK9 levels were independently associated with white blood cell counts among patients with stable CAD. ${ }^{40}$ The correlation between PCSK9 levels and white blood cell counts suggests a potential link between PCSK9 and chronic inflammation. The absence of PCSK9 has been found to protect against septic shock in mice. ${ }^{41}$ The effect of PCSK9 on inflammatory responses is further supported by another study, which reported that PCSK9 knock-down decreased cytokine expression and suppressed the inflammatory response through inhibition of nuclear factor- $\mathrm{kB}$ activation in macrophages. ${ }^{42-44}$

The current study found that lipoprotein (a) was an independent risk factor for SVGD. Many studies have shown that elevated plasma levels of lipoprotein (a) are associated with increased risk of cardiovascular disease. ${ }^{454}$ Lipoprotein (a) is one of the atherogenous lipoproteins and plays a crucial role in atherogenesis. The mechanism of lipoprotein (a) on atherogenesis is multifactorial and includes formation of atherosclerotic plaque, interference with plasminogen activation, disrupting endothelial function and promoting phospholipid oxidation. ${ }^{47}$

Several limitations should be considered in the interpretation of the results from the current study. First, this was a cross-sectional study, so temporality cannot be confirmed. It is impossible to determine whether the PCSK9 serum levels are influenced by late SVGD or if SVGD risk is affected by PCSK9 levels. Second, the risk factors were significantly different between participants with SVGD and those without SVGD, including time from CABG surgery, smoking, age at CABG operation and family history. To mitigate the potentially negative influence of these confounding factors, a multivariate logistic regression model was used to adjust for these risk factors. Last, the number of participants enrolled was relatively small and the participants with abnormal non-invasive test results or recurrent stable angina pectoris were enrolled in a single centre. Although the participants in the present study may not represent all patients operated with CABG, we expect that the relationship between PCSK9 serum levels and SVDG should persist in other populations, but with varying magnitudes of association. Thus, a larger multicentre prospective study is necessary to validate our results.

\section{CONCLUSIONS}

Our study is the first to identify the association between PCSK9 serum levels and late SVGD and the association persisted after adjustment for established cardiovascular risk factors. A multicentre prospective cohort study with large sample size should be conducted in the future to further research this relationship.

\section{Author affiliations}

${ }^{1}$ Logistics University of Chinese People's Armed Police Forces, Tianjin, China

${ }^{2}$ Cardiovascular Institute, Tianjin Chest Hospital, Tianjin, China

${ }^{3}$ Peking University Clinical Research Institute, Beijing, China

${ }^{4}$ Department of Cardiology, Tianjin Chest Hospital, Tianjin, China

${ }^{5}$ Independent Consultant, New York, USA

Acknowledgements The authors thank the following project staff for collecting the data: Jing Ma, Li-li Zhao and Ying Zhang from Tianjin Chest Hospital.

Furthermore, they thank the research staff for their efforts in the study. Last but not least, they thank the subjects for their participation.

Contributors JG, YL, J-YX, and MR contributed to the development of the study protocol. JG and YL were the principal investigators and managed the protocol. JG, $\mathrm{H}-\mathrm{BW}, \mathrm{KHR}$ and Y-ML were involved in the initial draft of the manuscript and writing it. GJ and $\mathrm{H}-\mathrm{BW}$ were involved in reviewing the manuscript. All authors read and approved the final manuscript.

Funding This research was funded by the Key Project of Scientific and Technological Support Plan of Tianjin in 2016 (no.: 16YFZCSY00800) and the Key Project of Healthcare Industry of Tianjin in 2015 (no.: 15KG128).

Competing interests None declared.

Patient consent Obtained.

Ethics approval The study protocol and informed consent form were approved by the institutional review board of Tianjin Chest Hospital and the study was carried out in accordance with good clinical practices.

Provenance and peer review Not commissioned; externally peer reviewed. Data sharing statement № additional data are available.

Open access This is an open access article distributed in accordance with the Creative Commons Attribution Non Commercial (CC BY-NC 4.0) license, which permits others to distribute, remix, adapt, build upon this work non-commercially, and license their derivative works on different terms, provided the original work is properly cited, appropriate credit is given, any changes made indicated, and the use is non-commercial. See:Ohttp://creativecommons.org/licenses/by-nc/4.0/.

\section{REFERENCES}

1. GBD 2013 Mortality and Causes of Death Collaborators. Global, regional, and national age-sex specific all-cause and causespecific mortality for 240 causes of death, 1990-2013: a systematic analysis for the Global Burden of Disease Study 2013. Lancet 2015;385:117-71.

2. Head SJ, Davierwala PM, Serruys PW, et al. Coronary artery bypass grafting vs. percutaneous coronary intervention for patients with three-vessel disease: final five-year follow-up of the SYNTAX trial. Eur Heart J 2014;35:2821-30.

3. Goldman S, Sethi GK, Holman W, et al. Radial artery grafts vs saphenous vein grafts in coronary artery bypass surgery: a randomized trial. JAMA 2011;305:167-74.

4. Kim FY, Marhefka G, Ruggiero NJ, et al. Saphenous vein graft disease: review of pathophysiology, prevention, and treatment. Cardiol Rev 2013;21:101-9.

5. Gosavi S, Mukherjee D. Natural History of Saphenous Vein Grafts. Interv Cardiol Clin 2013;2:251-8.

6. van Brussel BL, Voors AA, Ernst JM, et al. Venous coronary artery bypass surgery: a more than 20-year follow-up study. Eur Heart $J$ 2003;24:927-36.

7. White CW, Gobel FL, Campeau L, et al. Effect of an aggressive lipidlowering strategy on progression of atherosclerosis in the left main coronary artery from patients in the post coronary artery bypass graft trial. Circulation 2001;104:2660-5.

8. Hata M, Takayama T, Sezai A, et al. Efficacy of aggressive lipid controlling therapy for preventing saphenous vein graft disease. Ann Thorac Surg 2009;88:1440-4.

9. Ibanez B, James S, Agewall S, et al. ESC Guidelines for the management of acute myocardial infarction in patients presenting 
with ST-segment elevation: The Task Force for the management of acute myocardial infarction in patients presenting with ST-segment elevation of the European Society of Cardiology (ESC). European heart journal 2017:2018:119-77.

10. Gencer B, Mach F. Sweetless'n low LDL-C targets for PCSK9 treatment. Eur Heart J 2015;36:1146-8.

11. Shimada YJ, Cannon CP. PCSK9 (Proprotein convertase subtilisin/ kexin type 9) inhibitors: past, present, and the future. Eur Heart $J$ 2015;36:2415-24.

12. Levy E, Ben Djoudi Ouadda A, Spahis S, et al. PCSK9 plays a significant role in cholesterol homeostasis and lipid transport in intestinal epithelial cells. Atherosclerosis 2013;227:297-306.

13. Seidah NG, Awan Z, Chrétien M, et al. PCSK9: a key modulator of cardiovascular health. Circ Res 2014;114:1022-36.

14. Cohen JC, Boerwinkle E, Mosley TH, et al. Sequence variations in PCSK9, low LDL, and protection against coronary heart disease. $N$ Engl J Med 2006;354:1264-72.

15. Sabatine MS, Giugliano RP, Wiviott SD, et al. Efficacy and safety of evolocumab in reducing lipids and cardiovascular events. $N$ Engl $J$ Med 2015;372:1500-9.

16. Robinson JG, Farnier M, Krempf M, et al. Efficacy and safety of alirocumab in reducing lipids and cardiovascular events. $N$ Engl $J$ Med 2015;372:1489-99.

17. Leander K, Mälarstig A, Van't Hooft FM, et al. Circulating Proprotein Convertase Subtilisin/Kexin Type 9 (PCSK9) Predicts Future Risk of Cardiovascular Events Independently of Established Risk Factors. Circulation 2016;133:1230-9.

18. Cariou B, Guérin P, Le May $C$, et al. Circulating PCSK9 levels in acute coronary syndrome: Results from the PC-SCA-9 prospective study. Diabetes Metab 2017;43:529-35.

19. Li S, Guo YL, Xu RX, Rx X, et al. Plasma PCSK9 levels are associated with the severity of coronary stenosis in patients with atherosclerosis. Int J Cardiol 2014;174:863-4.

20. Alonso R, Mata P, Muñiz O, et al. PCSK9 and lipoprotein (a) levels are two predictors of coronary artery calcification in asymptomatic patients with familial hypercholesterolemia. Atherosclerosis 2016:254:249-53.

21. Shan L, Saxena A, McMahon R, et al. Coronary artery bypass graft surgery in the elderly: a review of postoperative quality of life. Circulation 2013;128:2333-43.

22. Parang P, Arora R. Coronary vein graft disease: pathogenesis and prevention. Can J Cardiol 2009;25:e57-e62.

23. Vlachopoulos C, Terentes-Printzios D, Georgiopoulos G, et al. Prediction of cardiovascular events with levels of proprotein convertase subtilisin/kexin type 9: A systematic review and metaanalysis. Atherosclerosis 2016;252:50-60.

24. Boyer M, Lévesque V, Poirier P, et al. Impact of a 1-year lifestyle modification program on plasma lipoprotein and PCSK9 concentrations in patients with coronary artery disease. J Clin Lipidol 2016:10:1353-61.

25. Chan DC, Pang J, McQuillan BM, et al. Plasma Proprotein Convertase Subtilisin Kexin Type 9 as a Predictor of Carotid Atherosclerosis in Asymptomatic Adults. Heart Lung Circ 2016;25:520-5.

26. Shapiro MD, Fazio S, Tavori H. Targeting PCSK9 for therapeutic gains. Curr Atheroscler Rep 2015;17:499.

27. Shapiro MD, Fazio S. From Lipids to Inflammation: New Approaches to Reducing Atherosclerotic Risk. Circ Res 2016;118:732-49.

28. Libby P, Ridker PM, Maseri A. Inflammation and atherosclerosis. Circulation 2002;105:1135-43.
29. Urban D, Pöss J, Böhm M, et al. Targeting the proprotein convertase subtilisin/kexin type 9 for the treatment of dyslipidemia and atherosclerosis. J Am Coll Cardiol 2013;62:1401-8.

30. Ridker PM, Rifai N, Rose L, et al. Comparison of C-reactive protein and low-density lipoprotein cholesterol levels in the prediction of first cardiovascular events. N Engl J Med 2002;347:1557-65.

31. Rahel BM, Visseren FL, Suttorp MJ, et al. Preprocedural serum levels of acute-phase reactants and prognosis after percutaneous coronary intervention. Cardiovasc Res 2003;60:136-40.

32. Ding Z, Liu S, Wang X, et al. Cross-talk between LOX-1 and PCSK9 in vascular tissues. Cardiovasc Res 2015;107:556-67.

33. Chan DC, Hamilton SJ, Rye KA, et al. Fenofibrate concomitantly decreases serum proprotein convertase subtilisin/kexin type 9 and very-low-density lipoprotein particle concentrations in statin-treated type 2 diabetic patients. Diabetes Obes Metab 2010;12:752-6.

34. Twisk J, Gillian-Daniel DL, Tebon A, et al. The role of the LDL receptor in apolipoprotein B secretion. J Clin Invest 2000;105:521-32.

35. Schulz R, Schlüter KD. PCSK9 targets important for lipid metabolism. Clin Res Cardiol Suppl 2017;12:2-11.

36. Teusink B, Mensenkamp AR, van der Boom H, et al. Stimulation of the in vivo production of very low density lipoproteins by apolipoprotein $\mathrm{E}$ is independent of the presence of the low density lipoprotein receptor. J Biol Chem 2001;276:40693-7.

37. Reyes-Soffer G, Pavlyha M, Ngai C, et al. Effects of PCSK9 Inhibition With Alirocumab on Lipoprotein Metabolism in Healthy Humans. Circulation 2017:135:352-62.

38. Poirier S, Mayer G, Benjannet S, et al. The proprotein convertase PCSK9 induces the degradation of low density lipoprotein recepto (LDLR) and its closest family members VLDLR and ApoER2. J Biol Chem 2008;283:2363-72.

39. Roubtsova A, Munkonda MN, Awan Z, et al. Circulating proprotein convertase subtilisin/kexin 9 (PCSK9) regulates VLDLR protein and triglyceride accumulation in visceral adipose tissue. Arterioscler Thromb Vasc Biol 2011;31:785-91.

40. Li S, Guo YL, Xu RX, Rx X, et al. Association of plasma PCSK9 levels with white blood cell count and its subsets in patients with stable coronary artery disease. Atherosclerosis 2014;234:441-5.

41. Walley KR, Thain KR, Russell JA, et al. PCSK9 is a critical regulator of the innate immune response and septic shock outcome. Sci Trans Med 2014;6:258ra143.

42. Tang Z, Jiang L, Peng J, et al. PCSK9 siRNA suppresses the inflammatory response induced by oxLDL through inhibition of NF- $\mathrm{kB}$ activation in THP-1-derived macrophages. Int $\mathrm{J}$ Mol Med 2012;30:931-8.

43. Giunzioni I, Tavori H, Covarrubias R, et al. Local effects of human PCSK9 on the atherosclerotic lesion. J Pathol 2016;238:52-62.

44. Tang ZH, Peng J, Ren Z, et al. New role of PCSK9 in atherosclerotic inflammation promotion involving the TLR4/NF-KB pathway. Atherosclerosis 2017;262:113-22.

45. Kardys I, Oemrawsingh RM, Kay IP, et al. Lipoprotein(a), interleukin-10, C-reactive protein, and 8-year outcome after percutaneous coronary intervention. Clin Cardiol 2012;35:482-9.

46. Ikenaga $\mathrm{H}$, Ishihara $\mathrm{M}$, Inoue I, et al. Usefulness of Lipoprotein (a) for predicting progression of non-culprit coronary lesions after acute myocardial infarction. Circ J 2011;75:2847-52.

47. Feng Z, Li HL, Bei WJ, et al. Association of lipoprotein(a) with longterm mortality following coronary angiography or percutaneous coronary intervention. Clin Cardiol 2017;40:674-8. 